\title{
Serum acute phase proteins in Dirofilaria immitis and Wolbachia seropositive cats
}

2017. Vol 19(6) 693-696

(C) The Author(s) 2016

Reprints and permissions:

sagepub.co.uk/journalsPermissions.nav DOI: $10.1177 / 1098612 \times 15625435$ journals.sagepub.com/home/jfms

(S)AGE

\section{Ana C Silvestre-Ferreira',2, Lisete Vieira ${ }^{1}$, Hugo Vilhena ${ }^{2,3,4}$, Jose J Cerón ${ }^{5}$, Asta Tvarijonaviciute ${ }^{5,6}$, José A Montoya-Alonso ${ }^{7}$, Elena Carretón ${ }^{7}$ and Josep Pastor ${ }^{6}$}

\begin{abstract}
Objectives The aim of this study was to characterise the response of acute phase proteins (APPs) in cats seropositive for Dirofilaria immitis and to its endosymbiont bacterium Wolbachia.

Methods The APPs serum amyloid A (SAA), haptoglobin (Hp) and ceruloplasmin (Cp) were measured in 25 seropositive cats and in 16 healthy seronegative cats.

Results SAA and Cp concentrations were significantly higher in animals with $D$ immitis seropositivity that exhibited clinical signs related to the disease, and $\mathrm{Hp}$ was elevated in all $D$ immitis-seropositive animals. There was no significant correlation between APPs and $D$ immitis or Wolbachia species antibody titres.

Conclusions and relevance An association between feline seropositivity to $D$ immitis and APP response was demonstrated. Increases in serum SAA and Cp concentrations were related to $D$ immitis-associated clinical signs, whereas $\mathrm{Hp}$ increased in all seropositive animals.
\end{abstract}

Accepted: 11 December 2015

\section{Introduction}

Feline dirofilariosis is caused by the intravascular parasite Dirofilaria immitis, which is transmitted by culicid mosquitoes. ${ }^{1}$ Infection with $D$ immitis is preventable; however, once an animal is infected, it can develop heartworm disease and potentially life-threatening complications. Contrarily to dogs, in cats right-sided heart failure and caval syndrome have been rarely documented. ${ }^{2}$ In cats, the majority of the immature worms that reach the caudal pulmonary arteries die. This produces a strong vascular and parenchymal inflammatory response approximately 3 months after infection, inducing clinical signs such as coughing, dyspnoea or intermittent vomiting. In chronic feline heartworm infection, the adult heartworms suppress the function of pulmonary intravascular macrophages, reducing the severity of the clinical disease. ${ }^{2-4}$ Clinical presentation varies from asymptomatic infections to chronic respiratory signs, sometimes accompanied by chronic vomiting and even acute death with no premonitory signs. ${ }^{2}$ Owing to the unspecific clinical presentation, the low worm burdens and the weak levels of circulating antigens, diagnosis can be challenging and relies on a combination of tests, such as serological and parasitological tests, thoracic radiography and echocardiography. 2,4

Although cats appear to be more resistant than dogs to adult $D$ immitis infection, feline dirofilariosis has been

1Department of Veterinary Sciences, University of Trás-os-Montes and Alto Douro, Vila Real, Portugal

${ }^{2}$ Animal and Veterinary Research Centre (CECAV), University

of Trás-os-Montes and Alto Douro, Vila Real, Portugal

${ }^{3}$ Department of Veterinary Medicine, Vasco da Gama University School Coimbra, Coimbra, Portugal

${ }^{4}$ Baixo Vouga Veterinary Hospital, Segadães-Águeda, Portugal 5 Interdisciplinary Laboratory of Clinical Analysis (Interlab-UMU),

Veterinary School, Campus of Excellence Mare Nostrum, University of Murcia, Espinardo, Murcia, Spain

${ }^{6}$ Department of Animal Medicine and Surgery, Autonomous University of Barcelona, Barcelona, Spain

${ }^{7}$ Research Institute of Biomedical and Health Sciences (IUIBS),

Faculty of Veterinary Medicine, University of Las Palmas de Gran Canaria, Arucas, Las Palmas, Spain

\section{Corresponding author:}

Ana C Silvestre-Ferreira DVM, PhD, Department of Veterinary Sciences, University of Trás-os-Montes and Alto Douro, 5000-801 Vila Real, Portugal

Email: aferreir@utad.pt 
diagnosed worldwide. Studies performed in canine endemic areas show high feline seroprevalences, indicative of infection risk in cats. 5,6

Many filarial species, including $D$ immitis, harbour intracellular Wolbachia species, an endosymbiont bacterium. ${ }^{1,7}$ The presence of circulating Wolbachia species antibodies and Wolbachia species antigens in tissues of infected animals have been associated with inflammation. ${ }^{8,9}$

Serum acute phase proteins (APPs) are considered highly sensitive biomarkers of inflammation that can be of use in the diagnosis, management and prognosis of a variety of clinical conditions. ${ }^{10,11}$ In the cat, serum amyloid A (SAA) is considered a major APP, increasing a few hours after the inflammatory stimulus and remaining elevated for as long as the inflammation persists; haptoglobin ( $\mathrm{Hp}$ ) and ceruloplasmin $(\mathrm{Cp})$ are considered moderate APPs as their increment is moderate and slower, returning to normal values with a more gradual decline. ${ }^{10}$

It could be postulated that combining APP and $D$ immitis seropositivity could increase the index of suspicion of $D$ immitis as the cause of clinical signs. This survey aims to study APP response in $D$ immitis-seropositive cats. For this purpose serum SAA, Hp and Cp concentrations were studied in seropositive cats which were divided into three groups: asymptomatic, symptomatic with clinical signs compatible with $D$ immitis and symptomatic with signs not compatible with $D$ immitis. The influence of $D$ immitis titres and Wolbachia endosymbiont antibody titres on APP concentrations was also assessed.

\section{Materials and methods}

The SAA, Hp and Cp levels were determined in the sera of 41 cats, obtained from previous research. The study was approved by the ethical committee of the Veterinary Medicine Service of University of Trás-os-Montes and Alto Douro and was carried out in accordance with the current European legislation on animal protection. All owners were informed and gave informed consent to permit the utilisation of the blood samples of their animals. Dirofilaria immitis-seropositive cats were identified using serological techniques for anti- $D$ immitis and antiWolbachia antibody detection, ${ }^{6}$ and all cats were tested with real-time polymerase chain reaction (PCR) for Hepatozoon felis, Hepatozoon canis, Leishmania infantum and agents of the genera Babesia, Ehrlichia, Anaplasma, Rickettsia and Mycoplasma. ${ }^{12}$ Of them, 25 cats were seropositive to anti-D immitis and anti-Wolbachia antibodies, and 16 were seronegative cats that were considered healthy on physical examination (control animals). All the included animals were negative to the PCR-tested agents.

Seropositive animals were classified as asymptomatic (group 1; $\mathrm{n}=9$ ); symptomatic with clinical signs related to $D$ immitis such as anorexia, weight loss, cough, dyspnoea and vomiting (group 2; $\mathrm{n}=12$ ); and symptomatic with clinical signs triggered by other diseases (renal disease, otitis, pyothorax, limb abscess) not related to $D$ immitis (group 3; $\mathrm{n}=4$ ). This classification was made by an experienced internist who was blinded to the APP data before assigning cats to the groups.

The SAA concentrations were determined by a human turbidimetric immunoassay (LZ-SAA; Eiken Chemical) performed on an automated analyser (Olympus AU2700; Olympus Diagnostica). ${ }^{13}$ Serum Hp concentrations were determined by the haemoglobin binding method with the use of a commercial kit (Tridelta Development). ${ }^{13} \mathrm{Cp}$ concentrations were determined by a method previously described. ${ }^{14}$ The Hp and Cp analyses were performed in a biochemical autoanalyser (Cobas Mira Plus multi-parametric autoanalyser; ABX Diagnostic). All the methods showed inter- and intra-assay imprecision lower than $15 \%$, and the dilution of the samples resulted in linear regression equations with a correlation coefficient close to 1 .

The normal distribution of the studied parameters was accessed by a Kolmogorov-Smirnov test. Because the parameters did not follow a normal distribution, a Kruskal-Wallis test was used to assess differences in concentrations between groups. Mann-Whitney posttests with type I error correction were used whenever statistically significant differences were observed. A Spearman correlation test was used to study a possible link between anti-D immitis and anti-Wolbachia antibodies, and APP. Statistical significance was set at $P<0.05$.

\section{Results}

The values of the measured APPs in all groups are shown in Table 1. Serum SAA and Cp concentrations were significantly higher in cats of group 2 (D immitis-seropositive animals that showed compatible clinical signs) when compared with group 1 or group 3 animals $(P<0.05)$. Group 3 animals (symptomatic animals with diseases not related to $D$ immitis) showed similar serum concentrations of SAA and Cp to those of the control cats. Hp was statistically significantly higher in seropositive cats (groups 1, 2 and 3) when compared with control animals $(P<0.05)$.

The anti-D immitis and anti-Wolbachia antibodies titres from group 1 (asymptomatic cats) and group 2 (cats showing clinical signs) did not show statistically significant differences in seropositivity. No statistically significant correlation was observed between antibodies anti- $D$ immitis or anti-Wolbachia titres and APP concentrations.

\section{Discussion}

The aim of the present work was to evaluate the APP profile in $D$ immitis-seropositive cats through the use of a major (SAA) and two moderate APPs ( $\mathrm{Hp}, \mathrm{Cp})$. 
Table 1 Anti-Dirofilaria immitis and anti-Wolbachia antibodies titres, and concentrations of serum amyloid A (SAA), haptoglobin (Hp) and ceruloplasmin (Cp) in healthy (control) and infected cats

\begin{tabular}{|c|c|c|c|c|c|}
\hline & Control & $\begin{array}{l}\text { Without clinical } \\
\text { signs } \\
\text { (group 1) }\end{array}$ & $\begin{array}{l}\text { Compatible clinical } \\
\text { signs } \\
\text { (group 2) }\end{array}$ & $\begin{array}{l}\text { Not related clinical } \\
\text { signs } \\
\text { (group 3) }\end{array}$ & $\begin{array}{l}\text { Groups } \\
\text { comparison } \\
\text { (Kruskal-Wallis } \\
P \text { value) }\end{array}$ \\
\hline$n$ & 16 & 9 & 12 & 4 & \\
\hline $\begin{array}{l}\text { Anti-D immitis } \\
\text { antibody } \\
\text { titres }\end{array}$ & ND & $0.757(0.556-0.891)$ & $0.811(0.708-0.969)$ & $0.797(0.701-0.903)$ & 0.347 \\
\hline $\begin{array}{l}\text { Wolbachia } \\
\text { titres }\end{array}$ & ND & $0.697(0.560-0.935)$ & $0.777(0.600-0.962)$ & $0.6635(0.623-0.724)$ & 0.217 \\
\hline SAA (mg/l) & $0.3(0.0-0.6)^{a}$ & $0.4(0.0-8.2)^{a}$ & $30.2(0.0-121.0)^{b}$ & $1.05(0.30-58.1)^{a}$ & 0.001 \\
\hline $\mathrm{Hp}(\mathrm{g} / \mathrm{l})$ & $1.51(0.35-2.30)^{a}$ & $2.94(1.17-10.00)^{b}$ & $3.31(1.97-15.40)^{\mathrm{b}}$ & $3.88(1.33-10.10)^{b}$ & $<0.001$ \\
\hline $\begin{array}{l}\mathrm{Cp}(\Delta \mathrm{abs} / \mathrm{min} \\
\left.\times 10^{-3}\right)\end{array}$ & $4.5(0.3-54.0)_{a}$ & $5.5(0.4-7.1)^{a}$ & $9.4(5.6-55.0)^{b}$ & $5.2(3.0-5.5)^{\mathrm{a}}$ & $<0.001$ \\
\hline
\end{tabular}

Data are mean (interquartile range). Cp results are reported as the change in absorbance per minute at $550 \mathrm{~nm}$. Controls were seronegative to $D$ immitis. All samples from the groups 1-3 were seropositive to $D$ immitis Different letters between parameters indicate statistical significance $(P<0.01)$ $\mathrm{ND}=$ not detected

The results obtained indicated that serum SAA was elevated only in cats showing clinical signs compatible with $D$ immitis infection (group 2). Similarly, previous reports found that SAA concentration increased significantly in the acute phase but returned rapidly to baseline levels after a cat's experimental infection with either Mycoplasma haemofelis or 'Candidatus Mycoplasma haemominutum'.${ }^{13}$ Furthermore, only C-reactive protein (CRP), a canine major $\mathrm{APP}, 10$ showed significant differences between asymptomatic and symptomatic dogs infected with $D$ immitis, 15 and in human medicine major APPs such as CRP, SAA and Hp were found to be significantly elevated in patients with active Wuchereria bancrofti and Brugia malayi infection (the lymphaticdwelling filariae disease). ${ }^{16}$

Pulmonary inflammation caused by $D$ immitis may account for the increase in serum SAA in group 2 animals. Previous reports described respiratory signs in cats as attributable to the death of both juvenile and adult worms within the pulmonary arteries. ${ }^{3}$ Although, there seems to be evidence that Wolbachia species produces a greater acute inflammatory response, worsening the bronco-reactivity associated with $D$ immitis seropositivity in cats, ${ }^{17}$ in our study Wolbachia titres were similar between clinically affected and non-affected $D$ immitispositive animals.

All groups of cats seropositive for $D$ immitis showed elevation in serum $\mathrm{Hp}$ concentration in comparison with seronegative healthy animals. However, in dogs naturally infected with $D$ immitis, Hp was not increased in positive animals and even decreased in those presenting microfilaraemia. We hypothesised a connection between this decrease and the possible presence of haemolytic anaemia; ${ }^{15}$ however, no signs of haemolytic anaemia were found in the cats of our study. The reason for the increased serum concentrations of $\mathrm{Hp}$ in the present study is unknown. Although Hp levels can increase during inflammation unrelated to heartworm infection and therefore is not specific to this disease, it could be postulated that if increased Hp concentrations are detected in cats living in endemic areas, $D$ immitis infection should be included in the list of differential diagnoses.

Increased serum Cp levels were only observed in seropositive cats with clinical signs related to $D$ immitis infection. Although $\mathrm{Cp}$ is also related to oxidative stress, ${ }^{10}$ current knowledge on the level variations in feline inflammatory conditions is limited. Substantial further investigation is needed in order to determine its potential utility in cats.

A major limitation of this study is that antibody titres only confirm $D$ immitis exposure - seropositive cats are not necessarily infected. In addition, studies involving a larger number of animals to determine the sensitivity and specificity and optimal cut-off points of the different APPs for the detection of animals with clinical signs would be desirable. In the present study no significant differences were observed between $D$ immitis and Wolbachia antibody titres and the presence/absence of clinical signs. Based on the results, it could be postulated that disease activity of $D$ immitis be assessed by using the APP profile combined with evaluation of antibody levels when clinical signs and complementary diagnostic examinations suggest heartworm infection. These data are in accordance with studies in Leishmania-infected dogs, which suggest that CRP concentration reflects disease activity better than antibody concentration. ${ }^{18}$ Also, the determination of Wolbachia species titres is not likely 
to be useful for disease activity assessment, as, in this study, there was no evidence that exposure to these bacteria was associated with worse clinical signs.

\section{Conclusions}

To our knowledge, this is the first study to evaluate comprehensively the APP response of cats seropositive for anti-D immitis and anti-Wolbachia antibodies. Although precautions should be taken, owing to the relatively low number of animals included, the results show an association between feline seropositivity for $D$ immitis and an $\mathrm{APP}$ response. Increases in SAA and $\mathrm{Cp}$ are related to $D$ immitis-associated clinical signs, whereas Hp increases in seropositive animals regardless of the presence or absence of clinical signs.

Acknowledgements We are grateful to all owners and veterinarians who kindly collaborated in this study.

Conflict of interest The authors declared no potential conflicts of interest with respect to the research, authorship, and/ or publication of this article.

Funding Financial support was provided by the programme 'Juan de la Cierva' of 'Ministerio de Economia y Competitividad', Spain, through a postdoctoral grant.

\section{References}

1 Sironi M, Bandi C, Sacchi L, et al. Molecular evidence for a close relative of the arthropod endosymbiont Wolbachia in a filarial worm. Mol Biochem Parasitol 1995; 74: 223-227.

2 Litster AL and Atwell RB. Feline heartworm disease: a clinical review. J Feline Med Surg 2008; 10: 137-144.

3 McCall JW, Genchi C, Kramer LH, et al. Heartworm disease in animals and humans. Adv Parasitol 2008; 66: 193-285.

4 Lee AC and Atkins CE. Understanding feline heartworm infection: disease, diagnosis, and treatment. Top Companion Anim Med 2010; 25: 224-230.

5 Montoya-Alonso JA, Carretón E, Corbera JA, et al. Current prevalence of Dirofilaria immitis in dogs, cats and humans from the island of Gran Canaria, Spain. Vet Parasitol 2011; 176: 291-294.
6 Vieira L, Silvestre-Ferreira AC, Fontes-Sousa AP, et al. Seroprevalence of heartworm (Dirofilaria immitis) in feline and canine hosts from central and northern Portugal. J Helmintol 2014; 14: 1-5.

7 Taylor MJ and Hoerauf A. Wolbachia bacteria of filarial nematodes. Parasitol Today 1999; 15: 437-442.

8 Morchón R, Ferreira AC, Martín-Pacho JR, et al. Specific IgG antibody response against antigens of Dirofilaria immitis and its Wolbachia endosymbiont bacterium in cats with natural and experimental infections. Vet Parasitol 2004; 125: 313-321.

9 Kramer L, Grandi G, Leoni M, et al. Wolbachia and its influence on the pathology and immunology of Dirofilaria immitis infection. Vet Parasitol 2008; 158: 191-195.

10 Cerón JJ, Eckersall PD and Martinez-Subiela S. Acute phase proteins in dogs and cats: current knowledge and future perspectives. Vet Clin Pathol 2005; 34: 85-99.

11 Eckersall PD and Bell R. Acute phase proteins: biomarkers of infection and inflammation in veterinary medicine. Vet J 2010; 185: 23-27.

12 Vilhena H, Martinez-Díaz VL, Cardoso L, et al. Feline vector-borne pathogens in the north and centre of Portugal. Parasit Vectors 2013; 6: 99.

13 Korman RM, Cerón JJ, Knowles TG, et al. Acute phase response to Mycoplasma haemofelis and 'Candidatus Mycoplasma haemominutum' infection in FIV-infected and non-FIV-infected cats. Vet J 2012; 193: 433-438.

14 Cerón JJ and Martínez-Subiela S. An automated spectrophotometric method for measuring canine ceruloplasmin in serum. Vet Res 2004; 35: 671-679.

15 Méndez JC, Carretón E, Martínez S, et al. Acute phase response in dogs with Dirofilaria immitis. Vet Parasitol 2014; 204: 420-425.

16 Anuradha R, George PJ, Pavan Kumar N, et al. Circulating microbial products and acute phase proteins as markers of pathogenesis in lymphatic filarial disease. PLOS Pathog 2012; 8: e1002749.

17 García-Guasch L, Caro-Vadillo A, Manubens-Grau J, et al. Is Wolbachia participating in the bronchial reactivity of cats with heartworm associated respiratory disease? Vet Parasitol 2013; 196: 130-135.

18 Martinez-Subiela S, Strauss-Ayali D, Cerón JJ, et al. Acute phase protein response in experimental canine leishmaniasis. Vet Parasitol 2011; 180: 197-202. 